\title{
Time-resolved temperature and velocity field measurements in gas turbine film cooling flows with mainstream turbulence
}

\author{
Michael Straußwald ${ }^{1} \cdot$ Christopher Abram ${ }^{2} \mathbb{D} \cdot$ Tobias Sander $^{1} \cdot$ Frank Beyrau $^{2} \cdot$ Michael Pfitzner $^{1}$
}

Received: 3 August 2020 / Revised: 8 October 2020 / Accepted: 21 October 2020

(c) The Author(s) 2020

\begin{abstract}
Gas turbine film cooling strategies must provide adequate cooling performance under high levels of mainstream turbulence. Detailed information about the structure, dynamics and transport of the cooling films is needed to understand the flow physics and develop suitable numerical simulation tools. Here, we study film cooling flows in a wind tunnel with mainstream turbulence generated by an active turbulence grid. Gas temperature and velocity fields are measured using a laser-imaging method based on thermographic phosphor tracer particles. By replacing the previously used tracer $\mathrm{BaMgAl}_{10} \mathrm{O}_{17}: \mathrm{Eu}^{2+}$ with $\mathrm{ZnO}$, significant gains in accuracy and precision could be achieved. The increased sensitivity $(\sim 1 \% / \mathrm{K})$ of $\mathrm{ZnO}$ led to a threefold improvement in the single-shot, single-pixel temperature precision to $\pm 5 \mathrm{~K}$. The smaller particle size $\left(d_{\mathrm{p}, \mathrm{v}} \sim 600 \mathrm{~nm}\right)$ and agglomerated nanoparticle structure also reduced the tracing response time to $\sim 5 \mu$ allowing accurate tracking of turbulent fluctuations approaching $10 \mathrm{kHz}$. Moreover, no uncertainty arising from multiple scattering effects were observed using $\mathrm{ZnO}$ particles in this enclosed wind tunnel geometry at an estimated average seeding density of $2 \times 10^{11}$ particles $/ \mathrm{m}^{3}$. Timeaverage, fluctuation and single-shot temperature-velocity fields are presented for two mainstream turbulence levels $\left(\bar{u}^{\prime} / \bar{u}_{\mathrm{m}}\right.$ $=7 \%$ and $14 \%$ ) and two momentum ratios ( $\mathrm{IR}=4.7$ and 9.3 ) at a fixed density ratio of 1.55 . These flow conditions produce a cooling jet which is detached from the surface. High main flow turbulence causes faster mixing with the surrounding hot gas, increasing the wall-normal spreading of the cooling jet. The instantaneous flow fields show that mainstream turbulence has a significant effect on the shear layer velocity fluctuations and consequently on the streamwise and wall-normal turbulent heat flux, which is derived from the simultaneously acquired temperature-velocity data. We found that high mainstream turbulence reduces the heat flux away from the wall, suggesting that mainstream turbulence can act to diminish cooling performance. Sets of instantaneous measurements recorded at a $6 \mathrm{kHz}$ repetition rate also reveal the dynamic interactions between the main flow turbulence and the cooling jet. These findings and the recorded data can be used to advance turbulence modelling for numerical simulations.
\end{abstract}

\section{Graphic abstract}

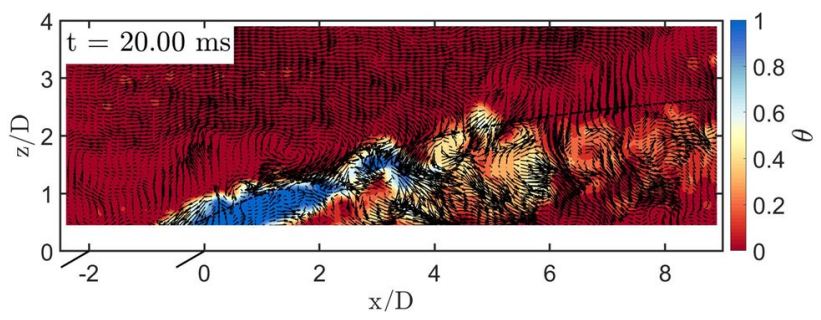

\section{Introduction}

Electronic supplementary material The online version of this article (https://doi.org/10.1007/s00348-020-03087-2) contains supplementary material, which is available to authorized users.

Extended author information available on the last page of the article
In modern gas turbine combustors, film cooling is an essential technology to reduce near-wall temperatures and protect liner and turbine blades from excessive heat loads. Cold air is injected through inclined holes or custom-designed inlet 
geometries with the aim of forming a protective layer of cold air between the hot gas and the wall. It is especially important that the protective layer remains closed so hot air cannot get in direct contact with the wall material. Although the majority of the film cooling studies focus on turbine blade applications, cooling of the combustor liner is a critical heat management aspect of gas turbine design, too. These two film cooling applications operate under different mainstream flow conditions, which influences the cooling film performance.

To understand the difference between the two applications, one has to consider the specific flow conditions in the liner and upstream of the turbine blades. The objective of the combustor liner is to provide optimal conditions for the combustion process. To ensure that fuel and air are sufficiently mixed before the combustion reaction starts, strong shear layers are generated which leads to high turbulence levels in the combustor. The combustion increases the flow temperature up to $2400 \mathrm{~K}$ (Bräunling 2015). Downstream of the combustion zone, the exhaust gas is prepared for the turbine. The flow is accelerated, which reduces its turbulence intensity, and it is mixed with cold air to reduce the temperature at the turbine inlet to the desired turbine entry temperature (Bräunling 2015). Overall, the reduced temperature and turbulence level of the flow at the turbine inlet leads to more homogeneous conditions for turbine blade cooling. This is in contrast with the more chaotic flow inside the combustor caused by the higher level of main flow turbulence, which can disrupt the cooling film and lead to increased gas temperatures adjacent to the wall (Martin and Thorpe 2012). The effective freestream turbulence level in the combustor can reach turbulence intensities above $100 \%$ and large vortices produce high turbulent length scales. For example, Martin and Thorpe (2012) found that the normalised turbulent length scale $\left(\Lambda_{\mathrm{D}}\right.$, based on the cooling hole diameter) of the freestream can exceed $\Lambda_{\mathrm{D}}=30$. These values are highly dependent on the geometry, the flow conditions and the location relative to the wall. Kakade et al. (2012), for instance, measured turbulence conditions in an enginescale annular combustor at isothermal conditions. They found near-wall turbulence intensities larger than $\bar{u}^{\prime} / \bar{u}_{\mathrm{m}}=$ $25 \%$ (here and throughout this article, the main flow velocity and the time-average velocity fluctuation are denoted by $\bar{u}_{\mathrm{m}}$ and $\bar{u}^{\prime}$, respectively); and turbulent length scales (normalised by the combustor radius) larger than $\sim 15 \%$. Even in the case of turbine blade cooling, Kohli and Bogard (1998) state that the freestream turbulence intensity of the incoming flow field can still exceed $\bar{u}^{\prime} \bar{u}_{\mathrm{m}}=20 \%$. Film cooling studies with realistic main flow turbulence levels are necessary to understand the flow dynamics and the influence on the film cooling performance.

Several prior experimental studies have considered higher turbulence main flow conditions for film cooling flows.
Kakade et al. (2012), for example, measured adiabatic effectiveness, heat transfer coefficients and net heat flux reductions downstream of effusion holes with $20^{\circ}$ injection angle and main flow turbulence intensities up to $22 \%$. They conclude that higher turbulence causes a greater lateral distribution of the coolant. For blowing ratios between $\mathrm{BR}=1.0$ and 1.5 , where the jet is already detached from the surface, this change in the flow field has a small positive effect not only on the laterally averaged adiabatic film cooling effectiveness but also on the net heat flux reduction. For small blowing ratios, which correspond to attached cooling jets, main flow turbulence has a negative effect on both quantities. At higher blowing ratios, the increase in the heat transfer coefficient is not compensated by a higher cooling effectiveness, resulting in higher heat fluxes than without the cooling film. These findings were confirmed by Martin and Thorpe (2012) using infrared surface thermometry and thermocouple gas temperature field measurements in the same test rig. Both studies attribute the positive effect of high turbulence to detached coolant being transported back towards the surface, thereby reducing the surface temperature. The positive effect ends once the film cooling effectiveness does not compensate the increased heat transfer coefficient due to the disturbed flow field. Martin and Thorpe further mention that the increased lateral distribution of coolant comes with a reduction in the surface coverage in streamwise direction. Finally, also they conclude that any coolant that is transported back after mixing with the main flow reduces the cooling effectiveness. Schroeder and Thole (2016) combined measurements of the adiabatic film cooling effectiveness with 4-kHz-PIV velocity field measurements for shaped holes. In this study, the main flow was varied between low $\left(\bar{u}^{\prime} / \bar{u}_{\mathrm{m}}=0.5 \%\right)$, medium (5.6\%) and high (13.2\%) turbulence intensity. They found that the increased freestream turbulence increases dilution and the lateral spreading of the jet. They further concluded that, at high blowing ratios, higher turbulence can also have a positive effect on the adiabatic effectiveness, which is consistent with the results of Kakade et al. (2012). Since the heat transfer coefficient was not measured in this study, the influence of mainstream turbulence on the net heat flux was not evaluated. Later, Schroeder and Thole (2017) carried out thermal field measurements using a thermocouple array. Again, they concluded that main flow turbulence increases lateral spreading and dilution of the jet, validating their prior findings. Further studies on increased main flow turbulence are available in the literature, e.g. Kadotani and Goldstein (1979), Kohli and Bogard (1998), Saumweber et al. (2003), Saumweber and Schulz (2012), Wright et al. (2010, 2011a, b, c; 2013) and Kingery and Ames (2015). Most of these studies focus on wall temperature measurements to determine adiabatic effectiveness or heat transfer coefficients.

Only some of these studies present pointwise gas velocity measurements (e.g. Kadotani and Goldstein 1979) or 
2D velocity field measurements (e.g. Wright et al. 2010, 2011b, c; 2013). These flow field data help to understand aerodynamic effects in the cooling air region and support the development and validation of computational fluid dynamics simulations. High-spatial resolution measurements of the scalar field using non-intrusive diagnostics are also of particular value, especially the gas temperature since temperature is a parameter that determines the heat transfer to the wall. In addition, to determine the turbulent heat flux, which can help to improve turbulence models (Ling et al. 2015a, b), it is necessary to measure temperature and velocity at the same point and at the same instant in time, which is a difficult task. Time-resolved imaging methods can also reveal the influence of main flow turbulence on the dynamics and thermal structure of the film cooling flow.

Therefore, our objective is to use an optical measurement technique based on thermographic phosphor tracer particles (Abram et al. 2018) to provide such high-speed planar simultaneous temperature and velocity data to compare the influence of well-characterised main flow turbulence for different film cooling flow conditions. In our previous work, we used this method to measure temperature and velocity fields at high spatial $(\sim 1 \mathrm{~mm})$ and temporal $(6 \mathrm{kHz})$ resolution (Schreivogel et al. 2016) (the so-called "thermographic PIV" technique). Since then we have improved the diagnostics with a more sensitive phosphor zinc oxide $(\mathrm{ZnO})$ and an optimised experimental setup. We have installed a turbulence grid to create more realistic mainstream turbulence levels for the examination of the influence of turbulence on cooling films. This is the first of several studies in an extended campaign which aim to understand the interaction of main flow turbulence and film cooling flows and its effect on heat transfer under a wide range of conditions and cooling hole geometries. In this paper, we describe the new experimental setup and examine the influence of turbulent mixing on the flow field and temperature distribution, and provide direct 2D measurements of the turbulent heat flux derived from simultaneously measured temperature-velocity data.

\section{Experimental setup and data acquisition}

\subsection{Wind tunnel setup}

A closed loop wind tunnel facility at Universität der Bundeswehr München was used for the film cooling experiments. The main flow is driven by a radial blower and can be electrically heated up to $T_{\mathrm{m}}=373 \mathrm{~K}$. Honeycombs and meshes reduce the turbulence of the main flow before acceleration through a 5:1 contraction nozzle to the final test section. Figure 1 left shows the test section module with the origin of the coordinate system indicated at the downstream edge of the film cooling geometry. The overall flow crosssection is $400 \mathrm{~mm} \times 150 \mathrm{~mm}$. An active turbulence grid is installed upstream of the test section (see Fig. 2). Depending on the operating mode, the turbulence grid can generate turbulence levels between $4.5 \%$ and $21.5 \%$ above the test plate and turbulent length scales between $\Lambda_{\mathrm{D}}=2$ and $\Lambda_{\mathrm{D}}=20$, respectively. The designs of the turbulence generator and the methods to characterize the flow are documented in Bakhtiari et al. (2018). The test plate is fitted with a boundary layer suction device at its leading edge. A trip wire is placed 80-mm downstream of the leading edge to generate a new boundary layer and a heater foil is used to form an approximately adiabatic temperature profile normal to the plate. A recess in the test plate accepts film cooling hole inserts with the appropriate test geometry.

Liquid nitrogen-cooled air is supplied to the film cooling holes through a plenum. The air is cooled to control the density ratio between main flow and cooling air $\left(\mathrm{DR}=\rho_{\mathrm{c}} / \rho_{\mathrm{m}}=T_{\mathrm{m}} / T_{\mathrm{c}}\right)$. Downstream of the test plate, the aspirated boundary layer flow is returned into the wind
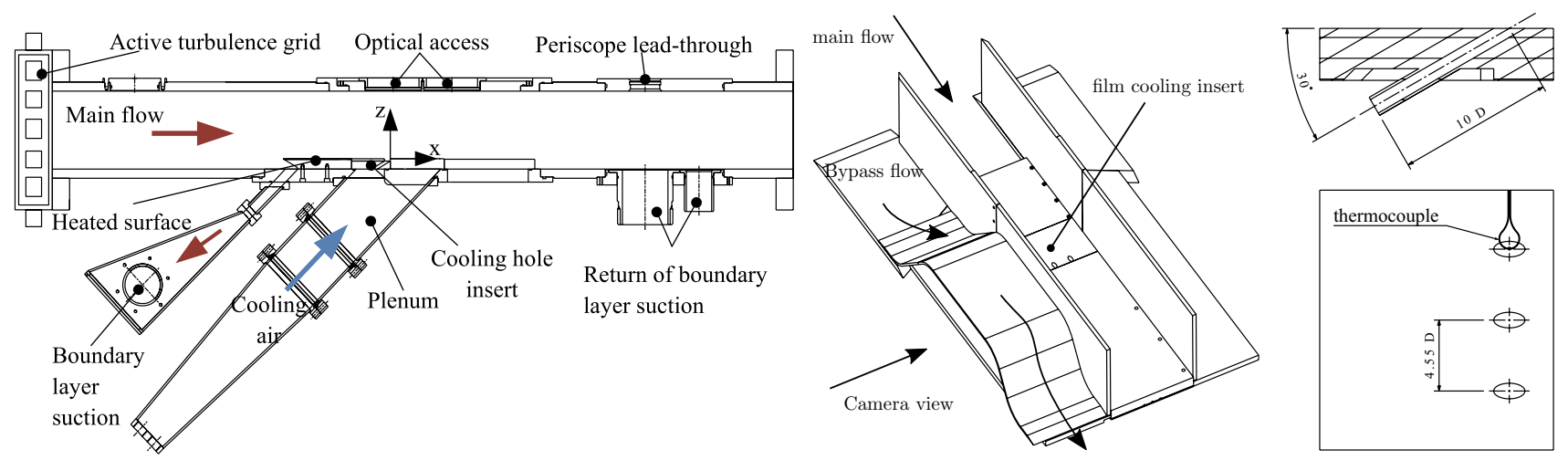

Fig. 1 Left-right: side view of the wind tunnel test section (Straußwald et al. 2018); 3D sketch of the modified test section; cooling hole geometry 

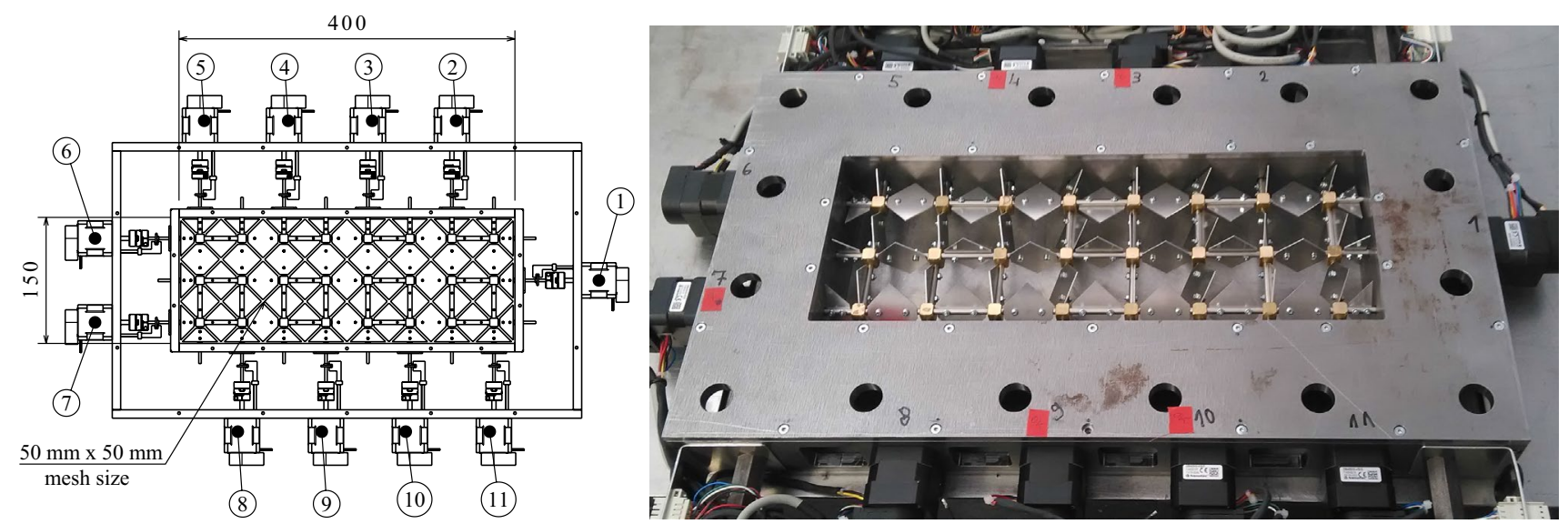

Fig. 2 Left: design drawing of the active turbulence grid (Strausswald et al. 2018). Right: photograph of the turbulence grid

tunnel. At the top wall and at the front side, optical access is provided through windows.

An important modification of the setup is the reduction of the amount of particle-seeded air between the camera and the light sheet. The reason is to avoid possible multiple scattering effects, meaning in this context that luminescence from one part of the flow is scattered by particles in another region, which scrambles the temperature information. Previous studies employing thermographic PIV using $\mathrm{BaMgAl}_{10} \mathrm{O}_{17}: \mathrm{Eu}^{2+}$ (BAM:Eu) particles noted measurement errors in film cooling flows (Schreivogel et al. 2016; Stephan et al. 2016) and round turbulent jets (Lee et al. 2016) arising from multiple scattering. A bypass geometry was mounted inside the test chamber (Fig. 1, middle). The upstream crosssection is now separated into three parts, to allow symmetric flow conditions in the central channel containing the film cooling test plate. The channel situated between the film cooling experiment and the camera view is designed such that the optical access to the central channel is unobstructed. This is achieved by a hump which leads the flow in the front channel above the region of interest.

\subsection{Flow conditions}

In this work, we focus on the results of a simple effusion hole geometry (Fig. 1, right) featuring one row with three uniformly spaced cooling holes. The holes have a diameter of $D=6 \mathrm{~mm}$ and are inclined at an angle of $\alpha=30^{\circ}$. The effusion hole geometry was equipped with a tube extension providing a total hole length of $10 \mathrm{D}$. This setup is used to prevent an inhomogeneous seeding density in the cooling jet which can be caused by a recirculation zone inside the hole. The effect on the flow condition has been discussed in a previous study (Abram et al. 2016).

Measurements were conducted at different nominal momentum ratios (IR $=D R \cdot \bar{u}_{\mathrm{c}}^{2} / \bar{u}_{\mathrm{m}}^{2}$, where $\bar{u}_{\mathrm{m}}$ and $\bar{u}_{\mathrm{c}}$ denote the average main flow and cooling air velocity, respectively) and main flow turbulence intensities. Flow temperatures were controlled using a 2-mm Pt100 resistance thermometer positioned in the main flow and a $0.1-\mathrm{mm}$ bare-wire type- $\mathrm{k}$ thermocouple for the cooling air. The cooling flow velocity was set and maintained with two mass flow controllers (Bronkhorst F-203AV-M50-RGD-44-V), while the mainstream velocity $\left(\bar{u}_{\mathrm{m}}=7 \mathrm{~m} / \mathrm{s}\right)$ was adjusted using the blower rotation speed and validated from the recorded PIV data. The nominal density ratio is $1.55\left(T_{\mathrm{c}}=240 \mathrm{~K}, T_{\mathrm{m}}=373 \mathrm{~K}\right)$. The velocity ratio was varied to produce two momentum ratios $I R=4.7$ and 9.3, and the turbulence grid was set to produce two main flow turbulence intensities of $\bar{u}^{\prime} / \bar{u}_{\mathrm{m}}=$ 7 and $14 \%$ ( $\bar{u}^{\prime}$ is the time-average of the velocity fluctuation). We evaluated the exact measured flow conditions at the time of each measurement sequence to determine the deviation in the density, velocity and momentum ratios and the turbulence intensities from the target nominal values. For comparison, all these boundary conditions are constant to within $\pm 5 \%$.

\subsection{Laser diagnostics}

Thermographic particle image velocimetry (thermographic PIV) uses luminescent particles seeded into the flow as a tracer for temperature and velocity. In our experiment, the particles are excited in a thin plane of the flow defined by a laser light sheet, and the temperature-dependent particle luminescence is imaged to determine the particle temperature via a two-colour ratio-based approach (Abram et al. 2018). At the same time, conventional velocity measurements are performed with standard PIV based on Mie scattering from the same particles generated using two laser pulses bracketing the excitation pulse and captured by an additional camera. The measurement accuracy relies on 
the rapid temperature and velocity equilibrium between the particle and gas phase. In this measurement campaign, we used micron-size zinc oxide thermographic phosphor particles (Sigma-Aldrich 96479, density $5.6 \mathrm{~g} / \mathrm{cm}^{3}$ ), which have two relevant features. First, the bandgap emission of $\mathrm{ZnO}$ has an inherently higher spectral shift with temperature in the range 300-500 K (Abram et al. 2015) compared to the $5 d-4 f$ luminescence emission of the BAM:Eu particles we used in our previous measurement campaign (Schreivogel et al. 2016), thereby improving the temperature sensitivity. Second, $\mathrm{ZnO}$ particles possess enhanced flow tracing properties due to the smaller particle size $(600 \mathrm{~nm}$ volumeequivalent diameter (Fond et al. 2018)) and specific morphology of $\sim 100 \mathrm{~nm}$ primary particles agglomerated into particles $\sim 1 \mu \mathrm{m}$ across [see electron microscope images in Abram et al. (2015)]. The relevant response time equivalent sphere-diameter of $380 \mathrm{~nm}$ can be calculated from the particle equation of motion in the Stokes regime using the hydraulic diameter (which determines the drag force) and the volume-equivalent diameter (which determines the inertial term), see Fond et al. (2018). Using this diameter, the 95\% response time of these particles to a step change in gas temperature or velocity is $\sim 5 \mu \mathrm{s}$, which represents more than an order of magnitude improvement in response time compared with $2.4 \mu \mathrm{m}$ BAM:Eu particles (Fond et al. 2012). The main flow and the cooling air were independently seeded with the $\mathrm{ZnO}$ particles using custom-made seeders.

Note that these $\mathrm{ZnO}$ particles are produced on an industrial scale using a gas phase process which could potentially introduce different spectroscopic properties for different batches. Using a dispersed particle characterisation system (Fond et al. 2019) we therefore verified that the absolute luminescence signal $\left(5 \times 10^{15}\right.$ photons per milligram of dispersed particles) was approximately the same for this batch of $\mathrm{ZnO}$ as for different batches of the same product purchased on prior occasions.

The main components of the measurement technique are shown in Fig. 3. A homogeniser and lenses were used to form the PIV light sheet from a double-pulse 532-nm diode-pumped Nd:YAG laser (Lee LDP-100 MQG) operated at $6 \mathrm{kHz}$ which is directed into the test section through the top window. The light sheet propagates perpendicular to the test plate and intersects the centerline of the central cooling hole. The pulse separation was $21 \mu$ s and the pulse energy was $\sim 1 \mathrm{~mJ}$. A frequency-tripled diode-pumped $\mathrm{Nd}$ :YAG laser at $355 \mathrm{~nm}, 6 \mathrm{kHz}$ repetition rate and $\sim 150$ ns pulse duration (Hawk HP-355-20-M with diode upgrade for maximum power output of $30 \mathrm{~W}$ at $6 \mathrm{kHz}$ repetition rate) is used as an excitation source for the phosphor particles. This light sheet was also formed using a homogeniser and lenses and had a final thickness of $1 \mathrm{~mm}$, corresponding to a laser fluence in the test section of $8 \mathrm{~mJ} /$ $\mathrm{cm}^{2}$. The beam was directed into the wind tunnel through a

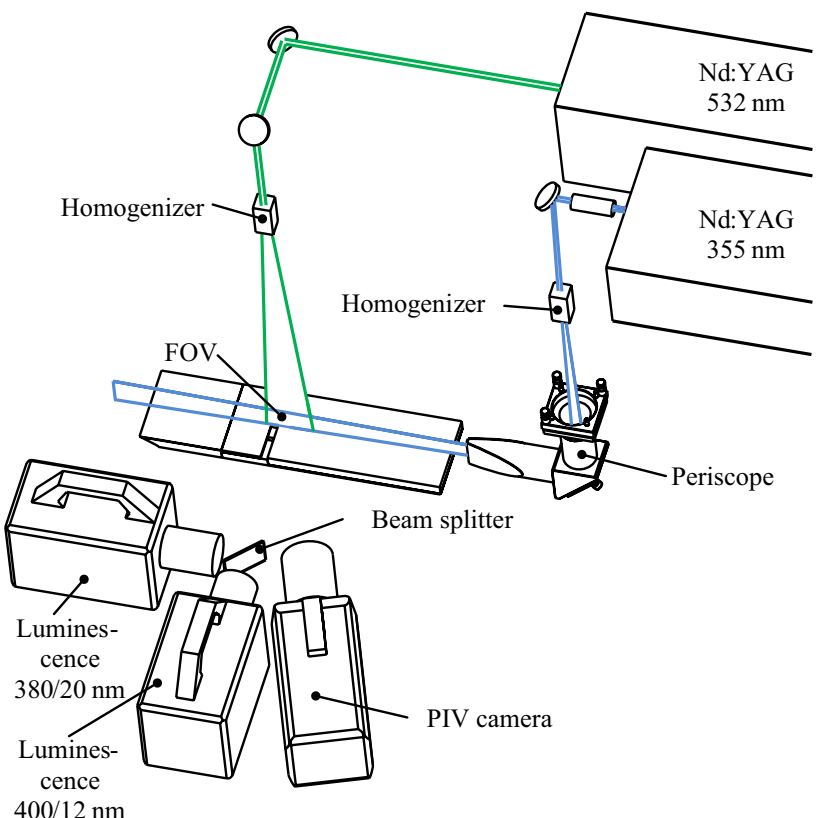

Fig. 3 Schematic of the hardware setup for thermographic PIV measurements using $\mathrm{ZnO}$, based on Schreivogel et al. (2016)

periscope, thereby preventing the laser light from directly impinging on the surface of the test plate. To maximise transmissivity for the UV luminescence signal, the side window for the optical access was made of fused silica (FQVIS 2, Hebo). Two Photron SA4 high-speed CMOS cameras are used for two-colour thermometry. The camera exposure time was set to $5.63 \mu \mathrm{s}$. However, note that the laser pulse duration (150 ns) and the luminescence lifetime of the semiconductor $\mathrm{ZnO}$ (1 ns) are both much shorter than the exposure time. Therefore, the duration of the laser pulse (effectively quasi-continuous excitation for $\mathrm{ZnO}$ ) defines the measurement duration which is $150 \mathrm{~ns}$. Luminescence signal is separated by a dichroic beamsplitter transmitting luminescence in the $390-420 \mathrm{~nm}$ range (see Fig. 4) and collected with $85-\mathrm{mm} \mathrm{f} / 1.4$ objective lenses. 1 " diameter 400/12 $\mathrm{nm}$ and 380/20 nm (notation central wavelength and full-width half-maximum transmission) bandpass filters for transmission and reflection cameras, respectively, are fixed between the camera chip and lens.

Mie scattered light was collected using a Phantom V710 camera with a $100-\mathrm{mm} f / 2$ lens and 540/15-nm bandpass filter and a Scheimpflug adapter. The Phantom camera was operated at $12 \mathrm{kHz}$ to generate PIV image pairs at a matching 6-kHz repetition rate using the standard high-speed PIV frame straddling method. The first 532-nm laser pulse is synchronous with the $355-\mathrm{nm}$ pulse to produce simultaneous velocity-temperature image pairs. 18,000 temperature and velocity fields were recorded for each 3 -s measurement series at a given flow condition. 


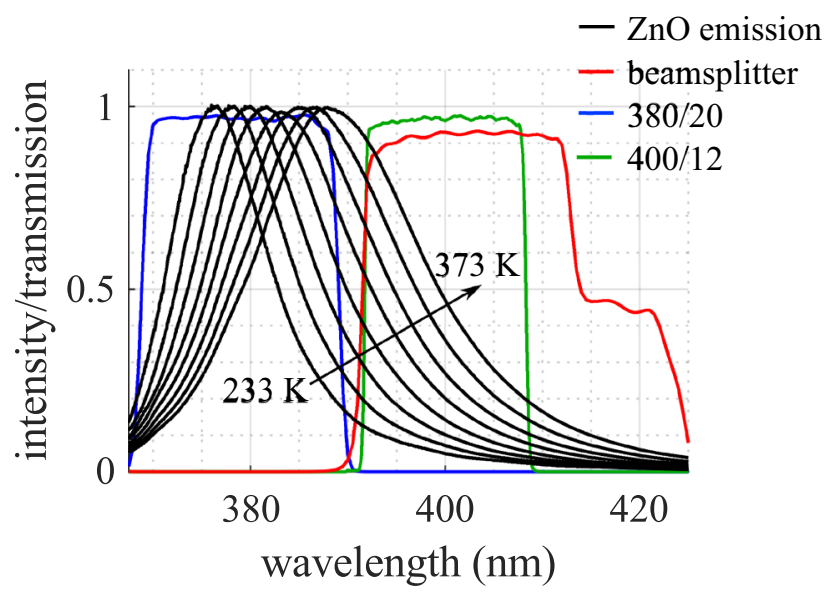

Fig. 4 Normalised $\mathrm{ZnO}$ emission spectra between 233 and $373 \mathrm{~K}$ at $20 \mathrm{~K}$ intervals. Filter and beamsplitter transmissivity curves are also shown

\subsection{Data processing}

For thermometry, image pairs were background subtracted, mapped using an image dewarping algorithm based on a dotmatrix target, binned ( 4 pixel $\times 4$ pixel) and smoothed using a 5 pixel $\times 5$ pixel moving average filter to a final nominal in-plane resolution of $1 \mathrm{~mm}$ (defined as the calculated object plane size of the binned and smoothed pixels), and divided by one another to generate intensity ratio fields. Single-shot intensity ratio images were also then divided by a time-average uniform room temperature intensity ratio field to correct for difference in collection angle and laser sheet profile, a point that we will return to later. Images were cropped below $z / D=0.45$ immediately adjacent to the wall, to ensure no error arising from background luminescence from deposited particles or the lower edge of the laser sheet could influence the interpretation of the temperature profiles.

To calibrate the thermometry system, the main flow and cooling air were controlled to various set temperatures. The thermocouple for the cooling air temperature is positioned at the side hole while the analyzed cooling jet emanates from the central hole (see Fig. 1). To verify that the temperature of the side hole exit can be used as a valid measurement for the central jet core temperature, simultaneous tests were also conducted with identical type-k thermocouples positioned both $20 \mathrm{~mm}$ inside and at the exit of the central hole, and a Pt100 probe (probe diameter of $1.5 \mathrm{~mm}$ ) was also placed at the side hole. For completeness, we report here that the difference between the thermocouples at these three positions was below $1 \mathrm{~K}$, and the difference with the Pt100 was no more than $2.5 \mathrm{~K}$, though these variations do not affect the data since normalised temperatures are used.

Mean intensity ratio values were calculated in these regions of known temperatures, namely the unaffected main flow and the core region of the detached cooling jet. A power law fit as shown in Fig. 5 was then used to reference the measured intensity ratios to temperature. The mean deviation of the fit to the raw data points recorded for various nominal temperatures on different days of the campaign is $\pm 6 \mathrm{~K}(1 \sigma, 4 \%$ of the normalised temperature). This value can be taken as the overall uncertainty of these measurements. Various causes for this uncertainty are evaluated below.

Particle Mie scattering image pairs were background subtracted and mapped to the same coordinate system as for the luminescence images. Velocity vector fields then were calculated using a multipass cross-correlation algorithm of the commercial software Davis 8 (LaVision) with an initial window size of 48 pixel $\times 48$ pixel and $50 \%$ overlap, and a final window size of 24 pixel $\times 24$ pixel and $75 \%$ overlap, resulting in a spatial resolution (defined as the calculated final vector spacing in the object plane) of $0.4 \mathrm{~mm}$. To calculate relevant temperature-velocity flow field statistics, the temperature data were interpolated onto the velocity fields.

Time-average fields were calculated from 6000 singleshot images for each in-plane location according to

$\bar{T}=\frac{1}{N} \sum_{i=1}^{N} T\left(t_{i}\right)$

and the temperature fields were normalised following $\theta=\left(T_{\mathrm{m}}-T\right) /\left(T_{\mathrm{m}}-T_{\mathrm{c}}\right)$. RMS fluctuation fields were calculated at each in-plane location using the expression
Fig. 5 Left: calibration data for $\mathrm{ZnO}$ particles recorded on different days and at different flow temperatures. Error bars based on deviation from the power law fit (see inset) at $240 \mathrm{~K}( \pm 7 \mathrm{~K}$, $1 \sigma)$ and $373 \mathrm{~K}( \pm 2 \mathrm{~K}, 1 \sigma)$ are also shown. The maximum deviation of a single datapoint to the fit is $10 \mathrm{~K}$. Right: sensitivity derived from the fit
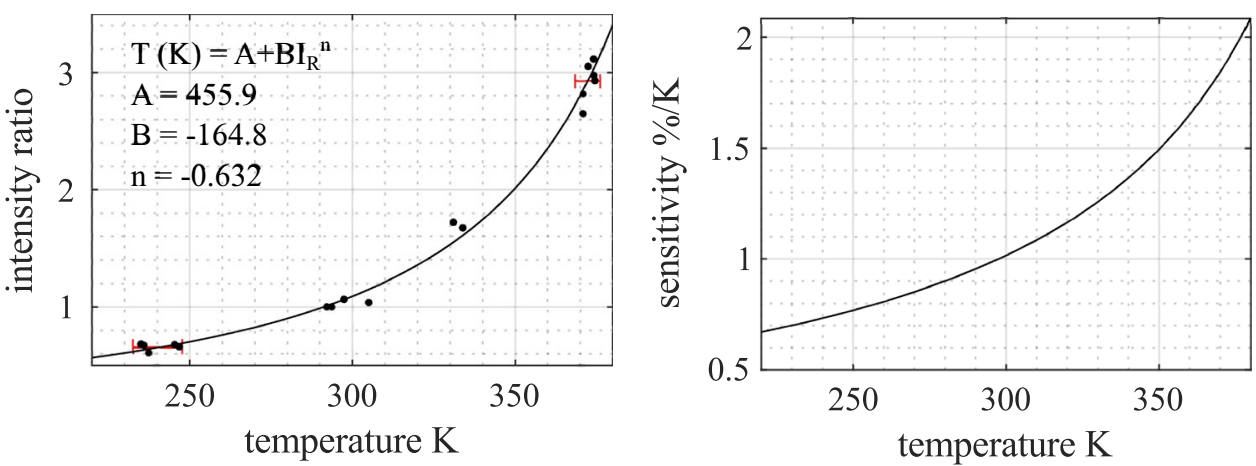
$\sigma_{T}=\sqrt{\frac{1}{N} \sum_{i=1}^{N}\left(T\left(t_{i}\right)-\bar{T}\right)^{2}}$,

and normalised by the difference between average main flow and cooling air temperature. For the velocity, similar expressions were used to calculate average flow fields and root mean square (RMS), using the average main flow velocity $\bar{u}_{\mathrm{m}}$ for normalisation. The turbulent heat flux was calculated from the product of the instantaneous velocity $u_{i}{ }^{\prime}$ and temperature $T_{i}{ }^{\prime}$ fluctuations for the streamwise $(x)$ and wallnormal $(z)$ direction. The time-averaged fields were then normalised according to $\overline{u_{x / z}^{\prime} T^{\prime}} /\left(\bar{u}_{\mathrm{m}}\left(T_{\mathrm{m}}-T_{\mathrm{c}}\right)\right)$.

\subsection{Uncertainty analysis}

To provide guidance for future experiments, here we examine three sources of systematic error which could contribute to the overall uncertainty in the calibration data, namely, multiple scattering, laser fluence effects, and uncertainty in the background subtraction.

First, as mentioned above, several thermographic PIV campaigns using BAM:Eu particles in similar jet in crossor co-flow geometries (Lee et al. 2016; Stephan et al. 2016), including our own (Schreivogel et al. 2016), have indicated that multiple scattering effects in the aerosol can lead to systematic temperature errors. Structured laser illumination was explored to tackle this issue in severe cases (Stephan et al. 2019; Zentgraf et al. 2017). In this study, to determine if multiple scattering from the large surrounding volume of hot particles influences the cooling flow temperature measurement in this test geometry, we performed experiments with and without main flow seeding. The seeding density is a parameter of specific importance. This was determined using the inverse of a method described in Fond et al. (2015); with the known emission intensity per particle of $\mathrm{ZnO}$ and the spectroscopic properties given in Fond et al. (2019) (accounting for laser fluence, gas temperature and excitation wavelength); and the diagnostic setup (including camera quantum efficiency, filter and objective lens transmission, magnification and light sheet thickness). Using this method, the main flow seeding density is estimated at $\sim 2 \times 10^{11}$ particles $/ \mathrm{m}^{3}$, with a factor 2 higher seeding density in the cooling jet $\left(\sim 4 \times 10^{11}\right.$ particles $\left./ \mathrm{m}^{3}\right)$ as determined from the Mie scattering intensity.

As shown in Fig. 6, the intensity ratio in the marked region of the jet core changed by only $~ 7 \%$ when seeding the main flow, a variation which is within the overall uncertainty reflected by the scatter in the calibration data $( \pm 6 \mathrm{~K}$, see Fig. 5). Therefore, we are convinced that in this geometry multiple scattering is not a significant issue when using $\mathrm{ZnO}$ at these seeding densities. This represents a significant improvement in comparison with our previous results
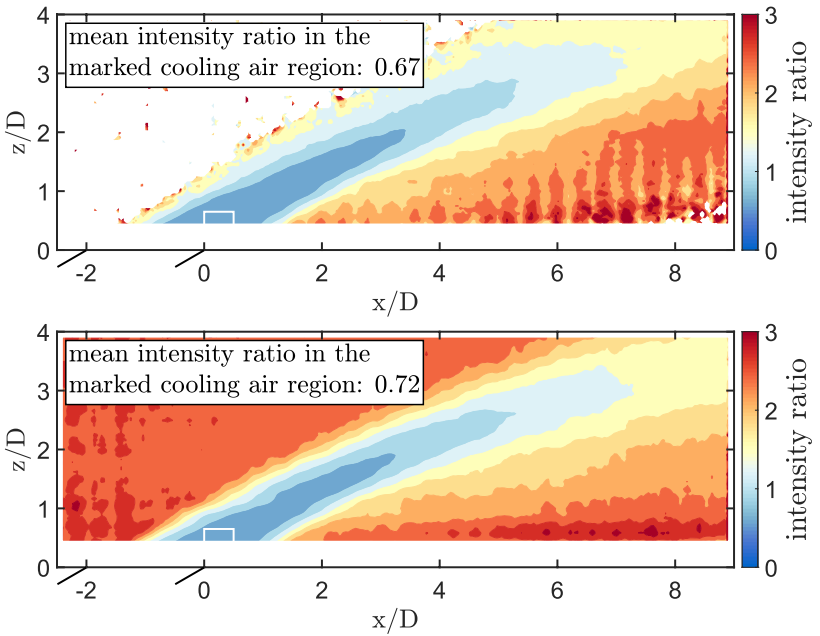

Fig. 6 Top: time-average intensity ratio field without main flow seeding; bottom: same, with main flow seeding at a calculated density of $\sim 2 \times 10^{11}$ particles $/ \mathrm{m}^{3}$

(Schreivogel et al. 2016) using BAM:Eu phosphor particles at seeding densities of a very similar magnitude $\left(\sim 3 \times 10^{11}\right.$ particles $/ \mathrm{m}^{3}$ ). In that study, a multiple scattering bias was identified which could only be mitigated with an in situ calibration method using the seeded main flow.

There are two possible factors contributing to the improvement in this measurement campaign. First, the higher absorption of $\mathrm{ZnO}$ relative to BAM:Eu leads to similar luminescence signal per particle (Fond et al. 2019), but, as discussed above, $\mathrm{ZnO}$ particles are clearly smaller than BAM:Eu particles and can be reasonably assumed to have a lower scattering cross-section. Therefore, for the same seeding density, the ratio of luminescence signal to scattered light can be expected to be higher for $\mathrm{ZnO}$ than BAM:Eu. Second, the modified test section geometry limits the number of particles on the optical path from light sheet to detection system, which could act to effectively reduce the parasitic influence of multiple scattering. In general, this is a promising result for this measurement technique. Still, additional systematic experiments in various geometries and illumination conditions in combination with multiple scattering modelling efforts are needed to better our understanding of these phenomena and to aid further improvements in the measurement design.

Second, a drawback of using $\mathrm{ZnO}$ tracer particles is that the measured temperature depends on the laser fluence. This is partly due to particle heating and partly due to a genuine photophysical effect at high power densities (Abram et al. 2015). These phenomena can cause measurement errors due to, for example, variation in the laser sheet profile or energy; or attenuation of the laser beam by the particles themselves as the beam propagates in the counter-streamwise direction. The laser power and homogenised sheet profile 
were extremely stable shot-to-shot and over the course of the measurement campaign. The seeding system was also relatively repeatable, with only $25 \%$ variation in the timeaverage Mie scattering signal between the measurement sequences presented here (note this is a measure unaffected by attenuation because the 532-nm light sheet enters the top of the test section and propagates only a short distance through the aerosol). Using the known optical properties of $\mathrm{ZnO}$ (Klingshirn 2007), and a Mie scattering and absorption calculation (Prahl 2018), the attenuation over the $\sim 300-\mathrm{mm}$ distance from the periscope to the measurement plane is estimated to be below $10 \%$ at $\sim 2 \times 10^{11}$ particles $/ \mathrm{m}^{3}$. Indeed, we found no correlation between the main flow seeding density (which could change the laser beam attenuation and, therefore, the laser fluence in the test section) and the measured main flow intensity ratio. These facts indicate that, for these experimental conditions of consistent, low-density seeding and stable laser operation, laser fluence effects are an insignificant source of uncertainty.

Third, a further source of systematic error is background subtraction. The background consists of the CMOS camera offset and also possible interference from particles deposited on the test plate and/or windows during the measurement. The test plate was carefully wiped clean between each measurement sequence. By subtracting time-average background images recorded before and after every measurement sequence from one another, differences below 1 count were identified, which leads to an error commensurate with the scatter in the calibration data in Fig. 5 left.

Besides the accuracy, the random uncertainty (precision), defined as the single-shot, pixel-pixel temperature error as determined from uniform temperature regions of the main flow at $373 \mathrm{~K}$, is $\pm 5 \mathrm{~K}$ ( $4 \%$ of the normalised temperature, $\theta=0.04)$ at $1 \mathrm{~mm}$ spatial resolution. This represents a threefold improvement compared to the previous use of the BAM:Eu phosphor. This is because the sensitivity of $\mathrm{ZnO}$ is $\sim 1 \% / \mathrm{K}$ at room temperature (Fig. 5), a fivefold improvement to the previous work.

Turning to the velocity measurement error, to determine the random error in the vector field images, an uncertainty calculation function based on the correlation statistics method (Wieneke 2015) available in the DaVis software was used. Average single-shot uncertainties in the main flow, cooling film and shear layer were estimated for an effusion hole test case at $\mathrm{IR}=9.3$. The random uncertainty is on average $0.5 \mathrm{~m} / \mathrm{s}$, corresponding to $\sim 10 \%$ of the instantaneous peak velocity fluctuation in the shear layer. The particle inertia will also damp measured velocity (and temperature) fluctuations. Following the formulae for the relative fluctuation velocities of the particle and fluid motions provided by Melling (1997), we estimate that turbulent velocity fluctuations up to $7 \mathrm{kHz}$ are tracked by these particles with less than 5\% error. At this frequency, the turbulent heat flux is underestimated by $6 \%$. Random and systematic errors are summarised in Table 1 (note the large datasets used here reduce the influence of random uncertainty in the mean flow field results to below 1\%).

\section{Results}

\subsection{Average flow field}

The cooling jets emanating from standard effusion holes are lifted off the surface, as shown in Fig. 7 for a momentum ratio IR $=4.7$ for $7 \%$ (left) and $14 \%$ (right) main flow turbulence. The upper image depicts the time-average normalised velocity field with the contour lines indicating the magnitude and arrows indicating the flow direction. The main flow enters the field of view horizontally from the left and the cooling air flow emanates from the hole graphically displayed in every plot. The lower contour plot shows the timeaverage-normalised temperature field. The high-temperature region in the wake of the jet indicates that hot air enters the near-wall region downstream of the jet. Previous PIV measurements in the same test rig revealed a broadening effect for already detached cooling jets at higher turbulence conditions (Straußwald et al. 2018). That study also suggested that cold air can be repeatedly pushed towards the surface which reduces the near-wall temperature and leads to an increased cooling efficiency for detached cooling jets, an effect also observed in Kakade et al. (2012). The velocity and temperature fields measured here confirm this finding, showing that while the average liftoff is almost identical, the jets spread out at high main flow turbulence levels, which can be explained by the jet mixing earlier due to the increased turbulent mixing intensity. The temperature plots indicate that the broadening effect does not reach the surface. Therefore, for this momentum ratio, the cooling efficiency is expected to be low for both turbulence levels.

Though not shown here for brevity, identical plots for the high momentum case (IR=9.3) show similar results for the average velocity and temperature fields, but the liftoff is slightly increased due to the higher momentum ratio. To

Table 1 Summary of error sources $( \pm 1 \sigma$ confidence $)$

\begin{tabular}{lll}
\hline Error source & Uncertainty & Notes \\
\hline Random velocity & $\sim 0.5 \mathrm{~m} / \mathrm{s}$ & - \\
Tracing error & $5 \%$ & Underestimate at $7 \mathrm{kHz}$ \\
Random temperature & $\pm 5 \mathrm{~K}$ & Determined at $373 \mathrm{~K}^{\mathrm{a}}$ \\
Systematic temperature & $\pm 6 \mathrm{~K}$ & $\mathrm{~b}, \mathrm{a}$
\end{tabular}



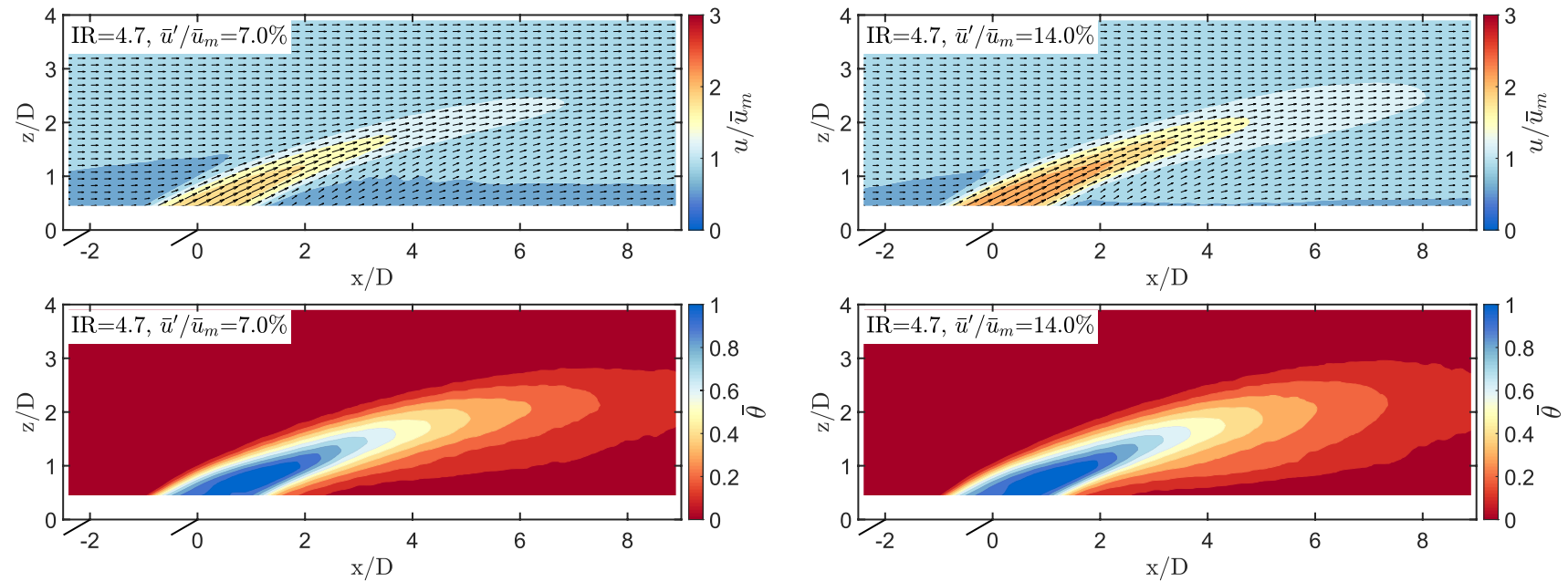

Fig. 7 Average velocity and temperature fields for momentum ratio IR=4.7. Left: main flow turbulence 7\%, right: main flow turbulence $14 \%$

better illustrate differences between the test cases at both momentum ratios and with different turbulence levels, the following figures compare wall-normal profiles of normalised temperature, temperature and velocity fluctuations, and turbulent heat flux at various downstream locations $(x / D)$.

Figure 8 shows the normalised temperature profiles for low $(I R=4.7)$ and high $(I R=9.3)$ momentum ratios, comparing low (7\%) and high (14\%) turbulence levels. The profiles near the jet exit $(x / D=0)$ hardly show any difference. Profiles for the low momentum ratio case confirm a slight broadening of the jet at high turbulence levels, matching the velocity fields shown above. For the higher momentum ratio, higher main flow turbulence causes a marginal but consistent increase in the temperature (decreased normalised

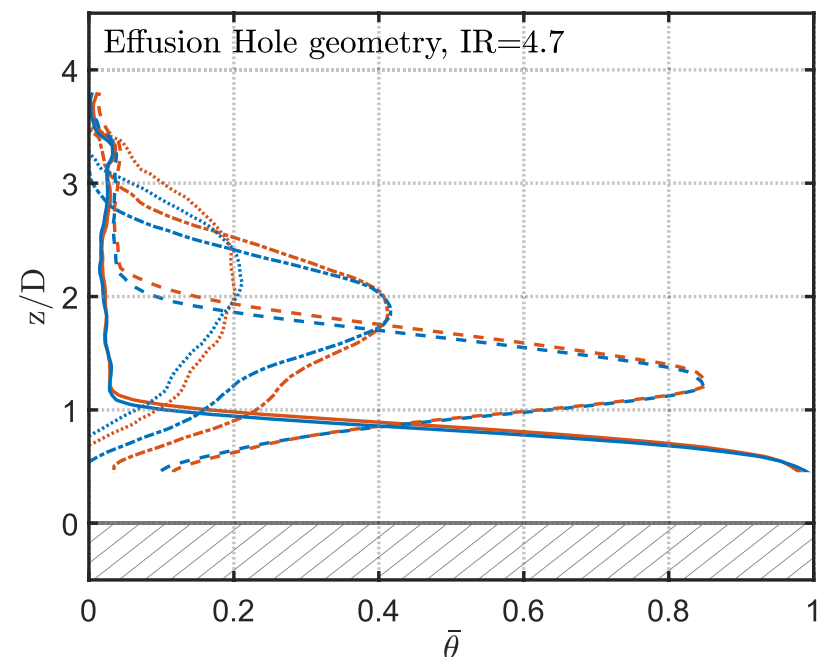

temperature) for all downstream positions, and a detectable shift in the peak of the normalised temperature toward the surface, indicating a lowering of the jet axis. Consistent with findings in several studies, e.g. Kakade et al. (2012), Martin and Thorpe (2012), Schroeder and Thole (2016, 2017) and Straußwald et al. (2018), these data confirm that higher turbulence levels increase the rate of mixing and broaden the shear layers.

\subsection{Fluctuation profiles}

The normalised velocity fluctuation profiles shown in Fig. 9 also indicate the influence of mainstream turbulence for both momentum ratios. The first peak at $x / D=0$

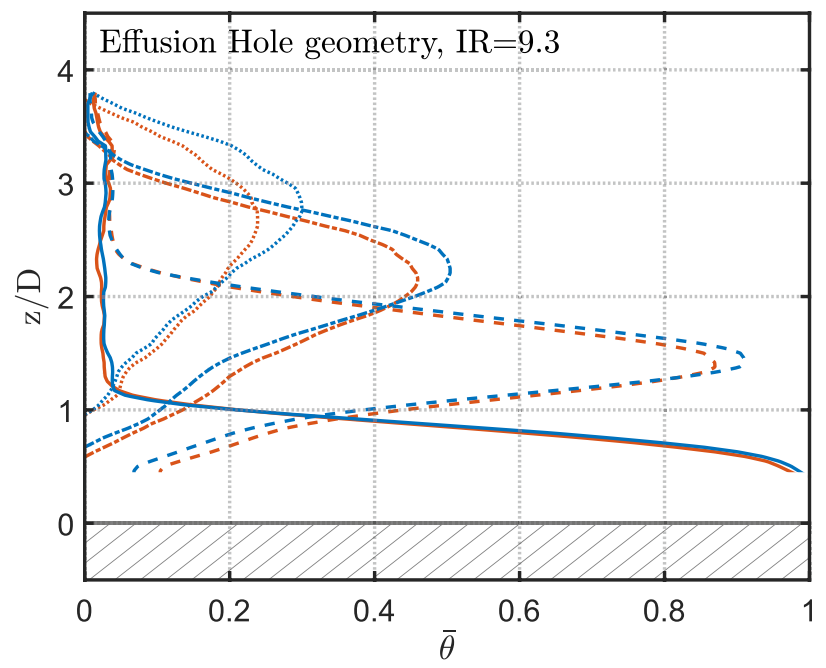

- $\bar{u}^{\prime} / \bar{u}_{m}=14.0 \%-\mathrm{x} / \mathrm{D}=0 \quad-\cdots-\mathrm{x} / \mathrm{D}=5$

- $\bar{u}^{\prime} / \bar{u}_{m}=7.0 \% \quad--\mathrm{x} / \mathrm{D}=2.5 \cdots \cdots \cdots \cdot \mathrm{x} / \mathrm{D}=7.5$

Fig. 8 Comparison of normalised average temperature $z$-profiles of low (7\%) and high (14\%) turbulence cases. Left: IR=4.7. Right: IR=9.3 

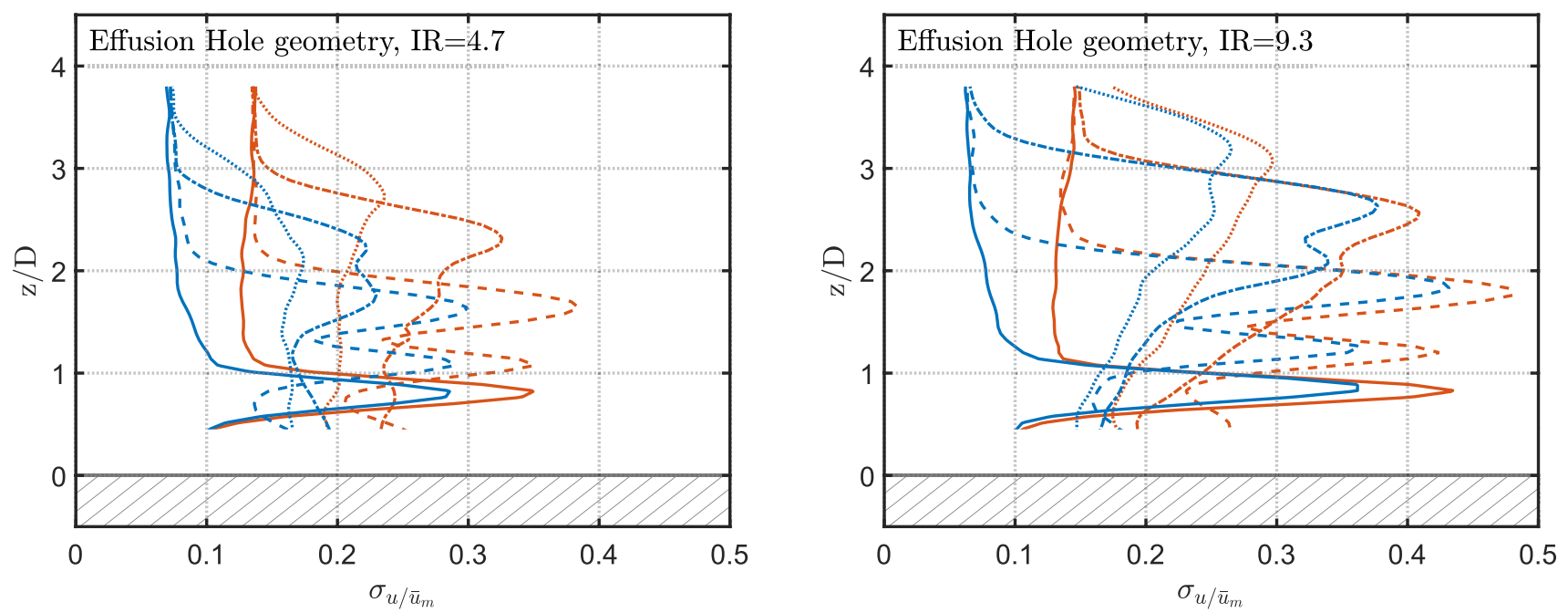

$\bar{u}^{\prime} / \bar{u}_{m}=14.0 \%-\mathrm{x} / \mathrm{D}=0 \quad-\cdots \times \mathrm{x} / \mathrm{D}=5$
- $\bar{u}^{\prime} / \bar{u}_{m}=7.0 \% \quad--\mathrm{x} / \mathrm{D}=2.5 \cdots \cdots \cdots \mathrm{x} / \mathrm{D}=7.5$

Fig. 9 Comparison of normalised velocity RMS wall-normal z-profiles of low (7\%) and high (14\%) turbulence cases. Left: IR=4.7. Right: $\mathrm{IR}=9.3$

reflects the fluctuations in upper part of the jet core where it penetrates the main flow, beyond which the profiles logically approach the main flow turbulence levels $(7 \%$ and $14 \%$ ). The wall-normal profiles demonstrate a prominent increase in velocity fluctuation along the streamwise direction from $x / D=0$ to $x / D=2.5$. Further downstream the fluctuations decay. For both momentum ratios, the higher main flow turbulence levels clearly increase the magnitude of the shear layer velocity fluctuations. The

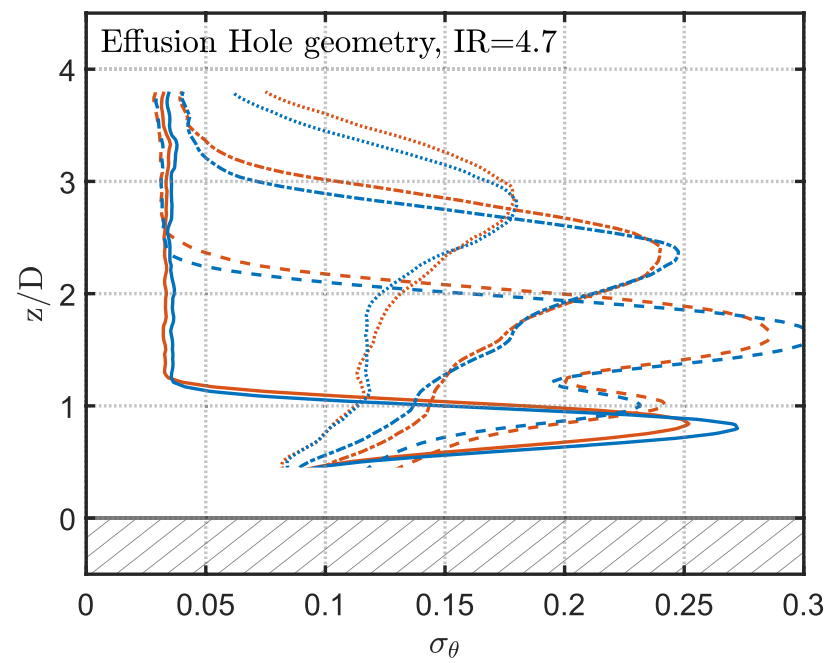

plots are consistent with the temperature fields, showing that higher turbulence causes earlier merging of the shear layers as indicated by the smoother profiles for $x / D>5$.

These findings can be contrasted with the temperature fluctuation profiles shown in Fig. 10. As expected, the streamwise positions of the temperature fluctuation peaks approximately mirror those of the velocity plots. Note that far from the wall, in the unaffected main flow, the fluctuation approaches $4 \%$ which is approximately the level

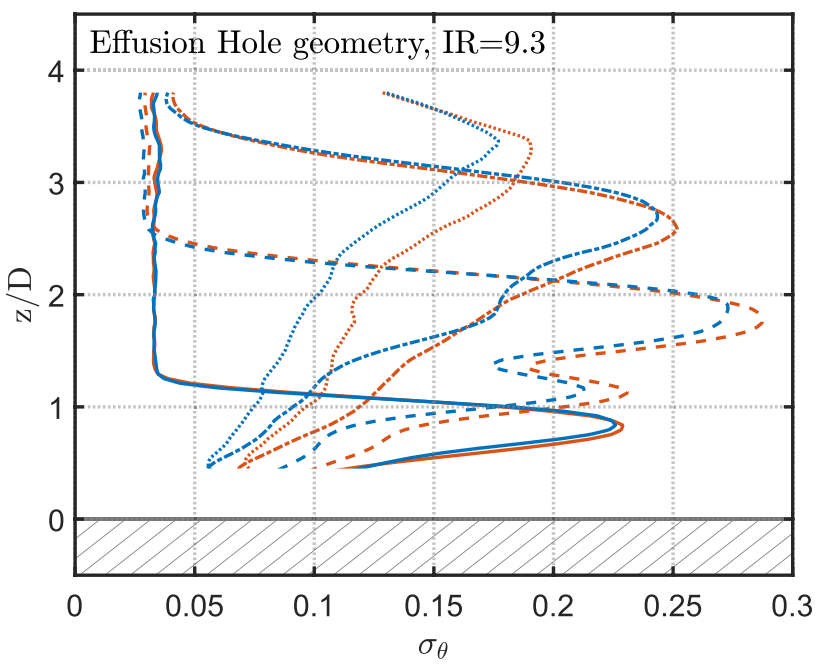

- $\bar{u}^{\prime} / \bar{u}_{m}=14.0 \%-\mathrm{x} / \mathrm{D}=0 \quad----\mathrm{x} / \mathrm{D}=5$

- $\bar{u}^{\prime} / \bar{u}_{m}=7.0 \% \quad--\mathrm{x} / \mathrm{D}=2.5 \cdots \cdots \cdots \cdot \mathrm{x} / \mathrm{D}=7.5$

Fig. 10 Comparison of normalised RMS temperature wall-normal $z$-profiles of low (7\%) and high (14\%) turbulence cases. Left: $\mathrm{IR}=4.7$. Right: IR=9.3 
of the measurement precision (see Table 1). Regarding the features of the temperature fluctuation profiles, while they appear slightly broadened, within the overall measurement uncertainty $(\theta= \pm 0.04)$ there is no pronounced increase in the magnitude of the temperature fluctuations with increased main flow turbulence. As the temperature of the mainstream is constant and independent of its turbulence level, the increased velocity fluctuations in the main flow do not lead to a significant difference in the magnitude of the temperature fluctuations within the shear layers.

\subsection{Turbulent heat flux}

The simultaneous measurement of instantaneous temperature and velocity fields provides all quantities necessary to calculate the turbulent heat flux. Figures 11 and 12 show the respective streamwise and wall-normal components of the turbulent heat flux for both momentum ratios and both turbulence levels. For all profiles, at $x / D=0$, a single peak is visible which can be identified as the upper shear layer. Downstream, at $x / D=2.5$, a second peak appears due to the lower shear layer. As indicated by the heat flux fields, for all flow conditions both the streamwise and wall-normal components of the heat flux in the upper shear layer are negative for all downstream locations. The overall magnitude of the heat flux is higher in the high momentum ratio case, a result of increased shear layer velocity fluctuations (see Fig. 9).

The negative streamwise heat flux (Fig. 11) is a finding consistent with previous studies in similar flows (Kohli and Bogard 1998; Schreivogel et al. 2016). Such observations contradict the gradient diffusion hypothesis which predicts a positive streamwise heat flux in the upper shear layer. This is true for all downstream locations and for both momentum ratios. Higher mainstream turbulence increases the magnitude of both the negative streamwise transport in the upper
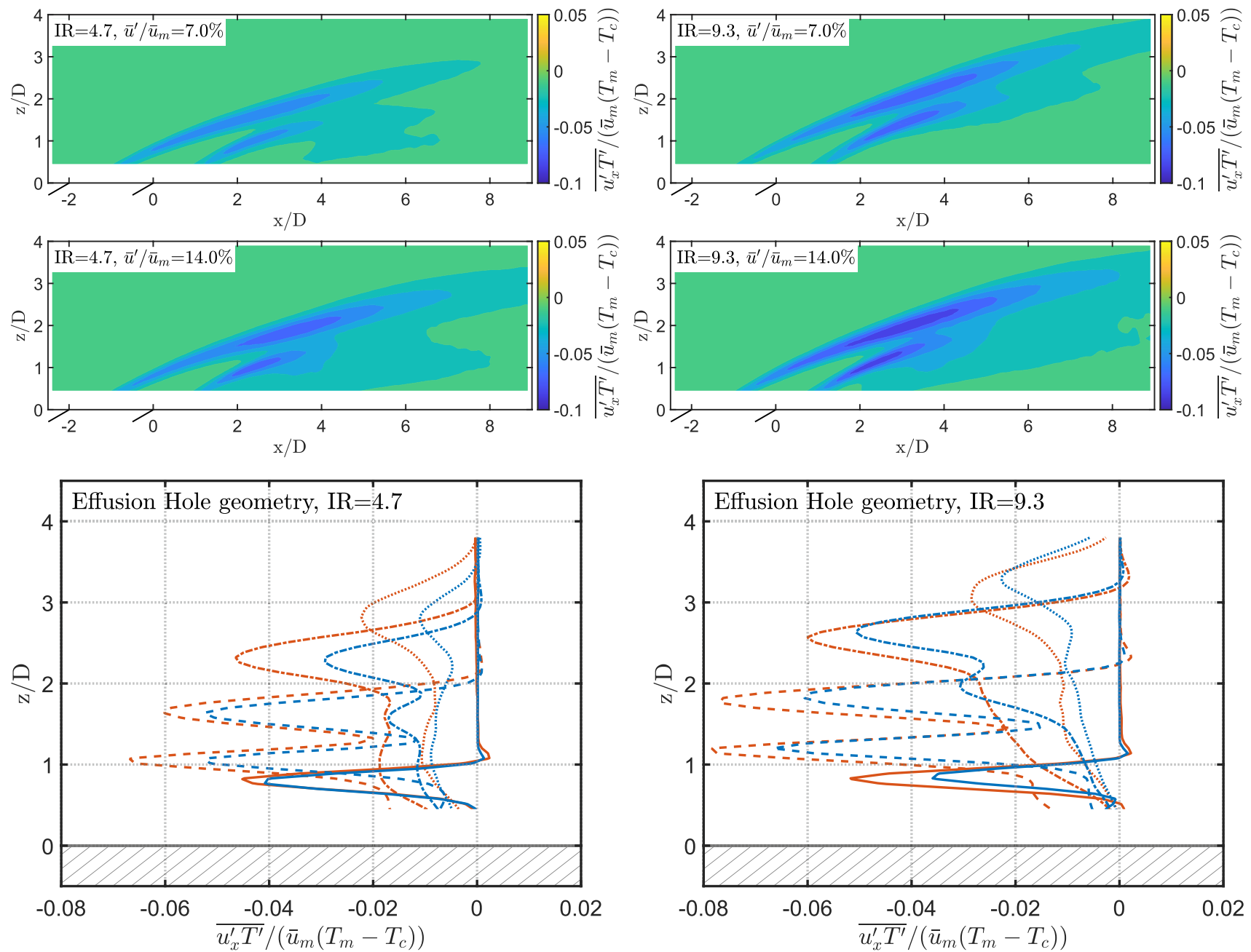

$$
\begin{aligned}
& \bar{u}^{\prime} / \bar{u}_{m}=14.0 \%-\mathrm{x} / \mathrm{D}=0 \quad-\cdots \mathrm{x} / \mathrm{D}=5 \\
& -\bar{u}^{\prime} / \bar{u}_{m}=7.0 \% \quad-\mathrm{x} / \mathrm{D}=2.5 \cdots \cdots \cdots \mathrm{x} / \mathrm{D}=7.5
\end{aligned}
$$

Fig. 11 Streamwise turbulent heat flux. Top panels show the heat flux fields for 7\% (upper) and 14\% (middle) main flow turbulence levels. The lower plots compare wall-normal profiles at indicated downstream locations. Left: IR =4.7. Right: IR =9.3 

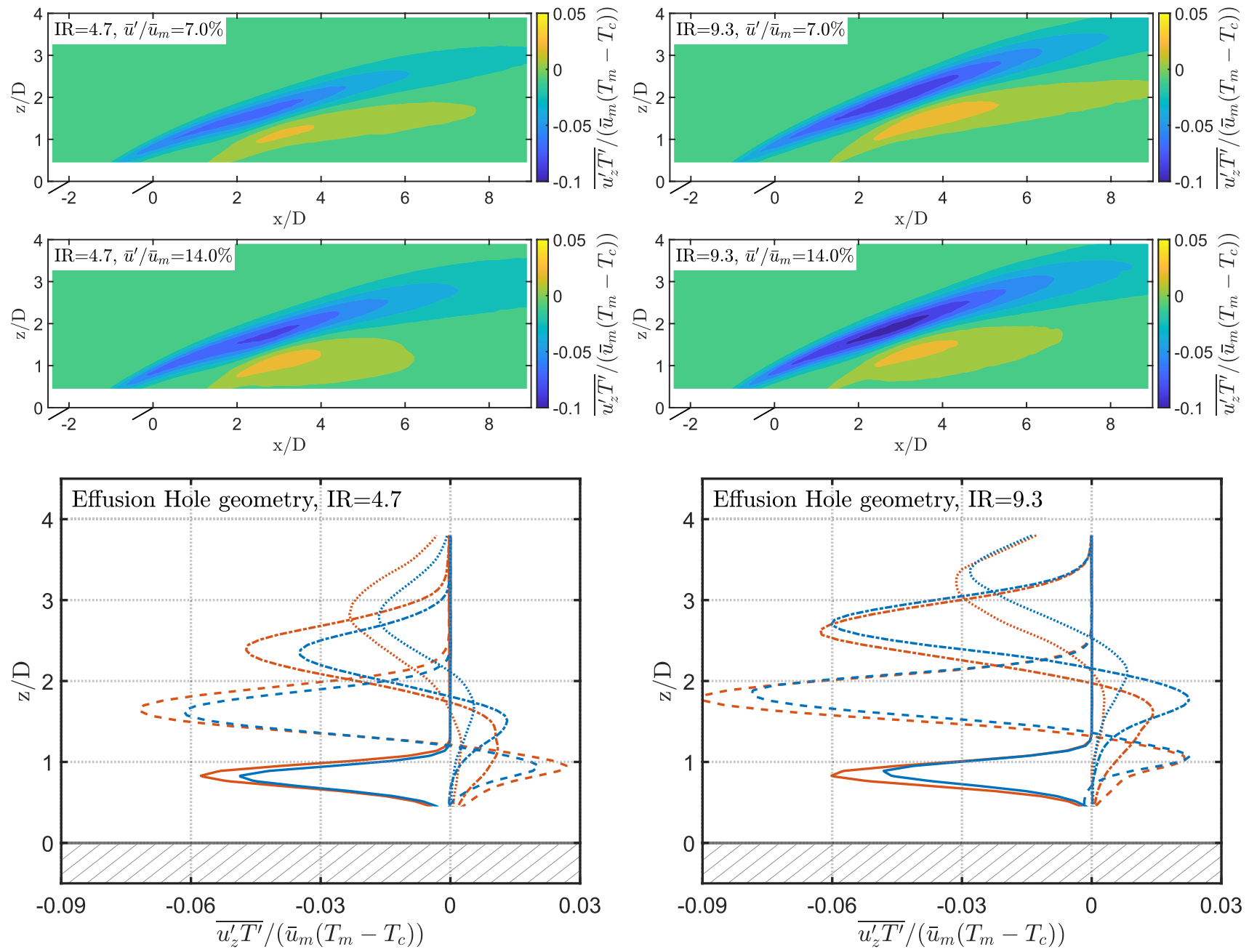

- $\bar{u}^{\prime} / \bar{u}_{m}=14.0 \%-\mathrm{x} / \mathrm{D}=0 \quad-\cdots \mathrm{x} / \mathrm{D}=5$

- $\bar{u}^{\prime} / \bar{u}_{m}=7.0 \%--\mathrm{x} / \mathrm{D}=2.5 \cdots \cdots \cdots \cdot \mathrm{x} / \mathrm{D}=7.5$

Fig. 12 Wall-normal turbulent heat flux. Top panels show the heat flux fields for 7\% (upper) and 14\% (middle) main flow turbulence levels. The lower plots compare wall-normal profiles at indicated downstream locations. Left: $\mathrm{IR}=4.7$. Right: $\mathrm{IR}=9.3$

shear layer, and the transfer of hot gas into the jet in the lower shear layer. Similar to the velocity fluctuation plots, we can see that the streamwise component of the heat flux in the lower shear layer is already smeared out when the mainstream turbulence is high. This shows that the distinct effect of the shear layer ends earlier than at lower main flow turbulence levels.

Referring to Fig. 12, the wall-normal component of the heat flux in the lower shear layer is positive. For both momentum ratios, beyond $x / D=2.5$, the effect of increased main flow turbulence is to reduce the magnitude of the wallnormal heat flux in the lower shear layer, while increasing the magnitude in the upper shear layer. At $x / D=5$ and further downstream the heat flux in the lower shear layer begins to approach zero for the case with high mainstream turbulence intensity. To summarise, in the upper shear layer, the magnitude of both components of the turbulent heat flux is increased by high main flow turbulence levels, while in the lower shear layer this is only true for the streamwise component. In combination, the wall-normal heat flux from the wake region upwards into the cooling jet is reduced, and more heat from the main flow is transferred downwards into the cooling jet. Therefore, overall, reduced heat transport away from the wall is expected for higher main flow turbulence levels.

These turbulent heat flux results can be compared with prior studies of wall heat transfer. For example, for cylindrical effusion holes, $\mathrm{DR} \sim 1$ and $\mathrm{BR}=2.0$ (corresponding to IR 4), high (22\%) mainstream turbulence was shown to increase the heat flux into the wall compared to low turbulence conditions (4\%), (Kakade et al. 2012) which is consistent with our above interpretation of the flow field data. 

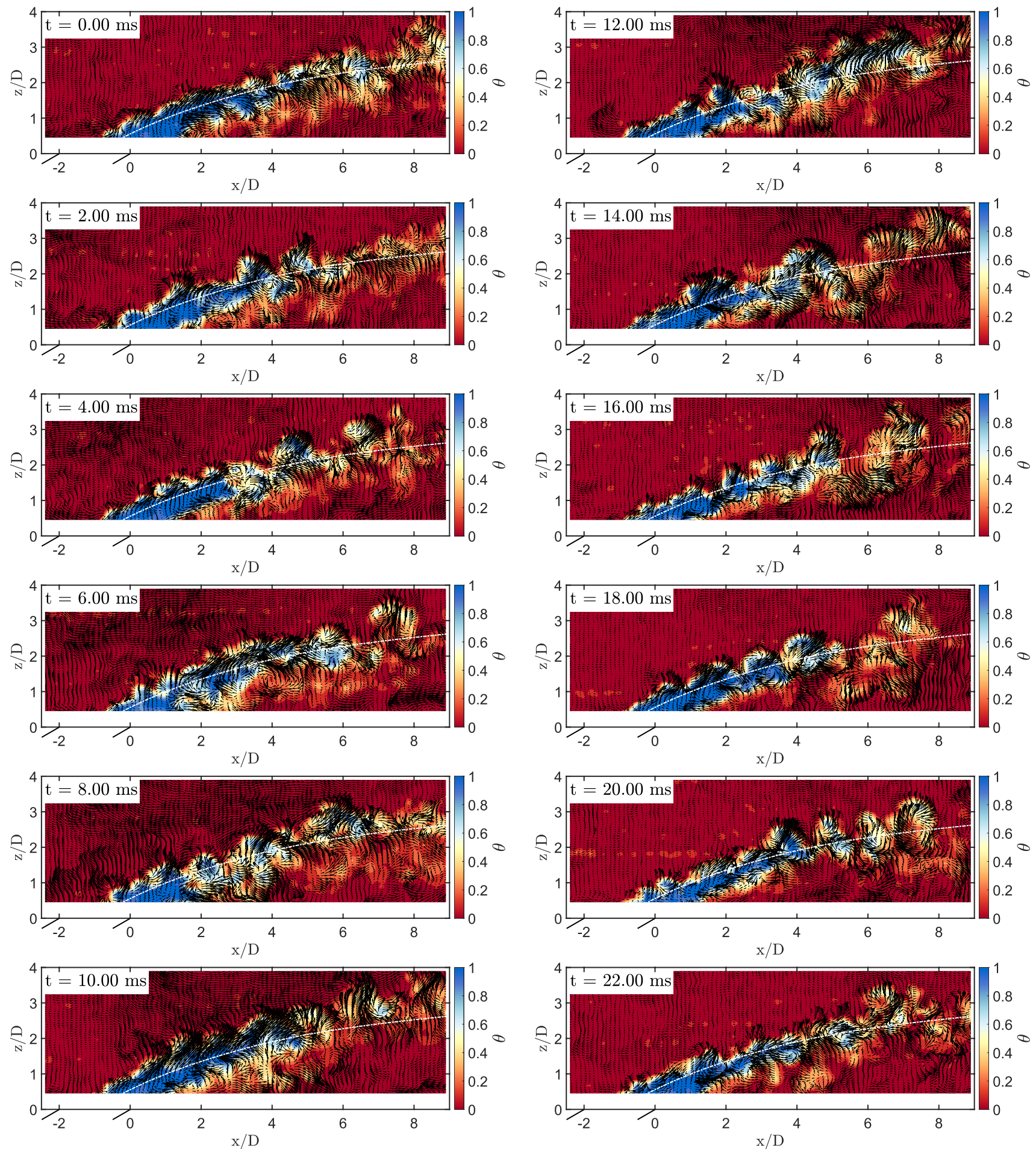

Fig. 13 Set of consecutive single-shot images sub-sampled at a rate of $500 \mathrm{~Hz}$ showing normalised temperature and velocity fluctuation fields for main flow turbulence $7 \%$, momentum ratio $I R=4.7$. The average central streamline is marked with a white dash-dotted line

Going forward, such simultaneous 2D temperature-velocity measurements provide a stringent benchmark for numerical simulations, and can directly link the effects of turbulent heat transport in the flow with the film cooling performance.

\subsection{Instantaneous flow field}

Videos of the instantaneous flow field have been produced and are provided online. The following sets of single-shot 

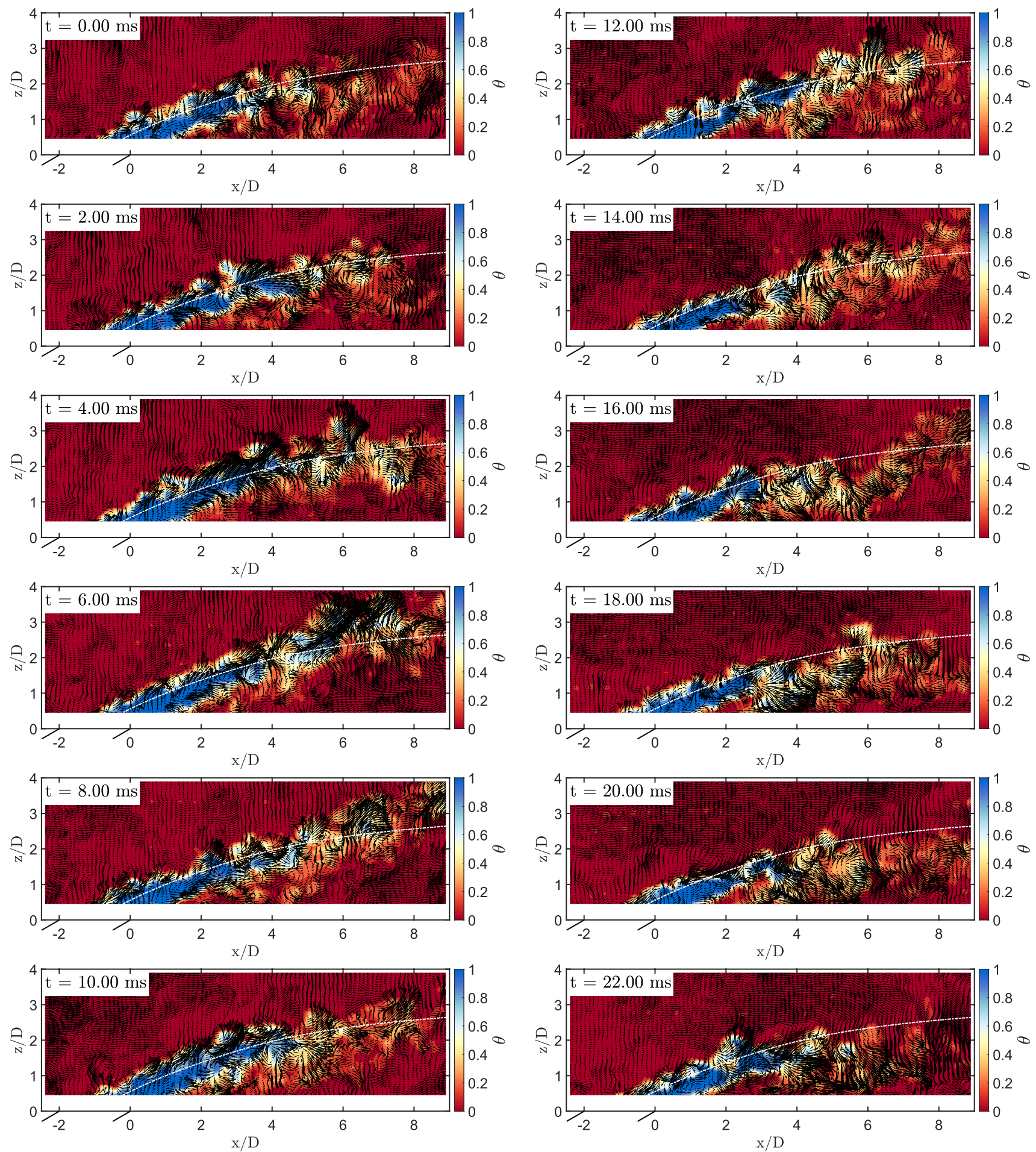

Fig. 14 Set of consecutive single-shot images sub-sampled at a rate of $500 \mathrm{~Hz}$ showing normalised temperature and velocity fluctuation fields for main flow turbulence $14 \%$, momentum ratio $\mathrm{IR}=4.7$. The average central streamline is marked with a white dash-dotted line

images are selected to reveal interesting flow features and differences between low and high main flow turbulence conditions. The images depict the normalised temperature field with the arrows indicating the velocity fluctuation. The sets consist of 12 consecutive images at a sub-sampled rate of $500 \mathrm{~Hz}$. The relative time stamp is indicated in every image.

Figure 13 shows images from the low turbulence case at $\mathrm{IR}=4.7$. The first image in the upper left corner provides 
a typical situation for the detached cooling jet which follows the average central streamline indicated with a white dash-dotted line. The temperature gradients between hot and cold regions are clearly visible immediately after the cooling hole. Further downstream, the cold air mixes with the surrounding hot gas. At $t=2 \mathrm{~ms}$, an eddy in the main flow enters the region upstream of the cooling jet. Following this disturbance through images from 4 to $12 \mathrm{~ms}$, it can be observed that the effect is to slightly lift the jet away from the surface. At $t=14 \mathrm{~ms}$, the disturbance passes the jet and the magnitude of the velocity fluctuation in the main flow is reduced. The jet promptly recovers and returns to its ordinary trajectory in the following images.

Figure 14 shows a set of images taken from the flow field measured at the same momentum ratio but for increased main flow turbulence (14\%). The images demonstrate how stronger fluctuations can affect the instantaneous flow field. At the beginning, the flow follows the average central streamline (again indicated with a white dash-dotted line). At this point in time, no unmixed coolant $(\theta \sim 1)$ is visible beyond $x / D \sim 3$, indicating the apparent jet penetration into the main flow is reduced either due to increased mixing or an out-of-plane motion, which suggests a strong impact on the jet at earlier times. Between $t=2 \mathrm{~ms}$ and $t=8 \mathrm{~ms}$, the apparent length of the jet recovers (hardly mixed coolant now visible beyond $x / D=4)$. This is accompanied with an increased lift off the surface which is driven by the strong velocity fluctuation in the wall-normal direction. At $t=10 \mathrm{~ms}$, an eddy with a high fluctuation velocity enters the field of view. In subsequent frames, it can be observed that the cooling jet is pushed back towards the centerline as the strong disturbance passes it, to the extent that at $t=16 \mathrm{~ms}$ the jet almost attaches to the surface. This shows that large deviations in the jet trajectory, and not simply a uniform broadening of the shear layers, are responsible for spreading of the jets at high mainstream turbulence levels.

Comparison of these two event sequences for two different main flow turbulence levels reveal subtle differences in the instantaneous flow field. Since the fluctuations in the low turbulence case are lower on average, the jet is apparently more stable even as a single vortex passes it. In the high turbulence case, however, changes in the flow field occur at a higher frequency, as the jet is deviated from its usual course in the in-plane direction and the apparent length is decreased due to mixing or an out-of-plane meandering motion. Under these conditions, the jet does not seem to recover for an extended period, and one can see that higher turbulence levels may lead to a more frequent occasions where hot air can reach the surface close to the holes. Of course, these flow features are highly variable, and the reader is invited to view the full sampling rate videos made available in the supplementary material and from which these images were selected, to examine the instantaneous flow fields for longer time periods. These and other flow features do occur in film cooling applications subject to high main flow turbulence, and the notion of a cooling film constantly protecting the wall from hot gases may not represent real flow conditions in an engine. The instantaneous time-resolved temperature and velocity field measurements enable the identification of differences of these flow characteristics for low and high turbulence conditions.

\section{Conclusions}

Simultaneous planar temperature-velocity fields were measured in film cooling flows emanating from $30^{\circ}$ inclined cylindrical effusion holes using a laser diagnostic technique based on thermographic phosphor particles. An active turbulence grid was added to the wind tunnel facility to provide mainstream turbulence levels relevant to gas turbine combustors. Improvements to the experimental setup are discussed. We achieve a temperature sensitivity of $1 \% / \mathrm{K}$ using $\mathrm{ZnO}$ phosphor particles, improving the single-shot single pixel temperature precision to $\pm 5 \mathrm{~K}$; and the small $(600 \mathrm{~nm})$ size and agglomerate morphology of the particles leads to improved flow tracing. Furthermore, no influence of multiple scattering was observed with $\mathrm{ZnO}$ particles in this geometry at an estimated average seeding density of $3 \times 10^{11}$ particles $/ \mathrm{m}^{3}$.

The influence of main flow turbulence (between $\bar{u}^{\prime}$ $/ \bar{u}_{\mathrm{m}}=7 \%$ and $\left.14 \%\right)$ on detached cooling jets were examined at two momentum ratios ( $\mathrm{IR}=4.7$ and 9.3). Average temperature and velocity fields indicate that the jet spreads out in the wall-normal direction. Examination of the timeresolved fields suggests that this is due to velocity fluctuations in the main flow lifting the jet off the surface and pushing it back. This leads to higher mixing rates of the cooling air with the surrounding flow. The fluctuation data further reveal increased shear layer fluctuations without an accompanying increase in the temperature fluctuations because the temperature of the mainstream is constant and independent of its turbulence level. Profiles of the turbulent heat flux indicate that higher levels of mainstream turbulence lead to an increase in the heat flux magnitude, except for the lower shear layer in the wall-normal direction, which reduces the heat flux away from the wall and suggests that mainstream turbulence can act to diminish cooling performance. The $\mathrm{kHz}$ sampling rates reveal additional dynamic interactions between the main flow and the cooling jet. Continuous fluctuations in the main flow arising due to higher turbulence levels cause the jets to deviate from their average position and cause higher oscillations in the jet trajectory. Future work will concentrate on lower momentum ratios and alternative geometries and examine in particular an improved 
trench geometry developed by Schreivogel et al. (2014). This collection of results will also provide a benchmark for comparison to numerical simulations.

Author contributions MS and MP conceived of the film cooling study. MS, CA and TS designed and performed the experiments. MS processed the experimental data. MS, CA, MP and TS discussed and analyzed the results. MS and CA wrote the manuscript. All the authors commented on and approved the final manuscript.

Funding Open Access funding enabled and organized by Projekt DEAL. The work was funded by Deutsche Forschungsgesellschaft (DFG, project number PF 443/7-1).

Availability of data and material Additional videos of the cooling jets for momentum ratio $I R=4.7$ and each main flow turbulence level are available as supplementary material.

\section{Compliance with ethical standards}

Conflict of interest The authors declare no conflict of interest.

Code availability Not applicable.

Open Access This article is licensed under a Creative Commons Attribution 4.0 International License, which permits use, sharing, adaptation, distribution and reproduction in any medium or format, as long as you give appropriate credit to the original author(s) and the source, provide a link to the Creative Commons licence, and indicate if changes were made. The images or other third party material in this article are included in the article's Creative Commons licence, unless indicated otherwise in a credit line to the material. If material is not included in the article's Creative Commons licence and your intended use is not permitted by statutory regulation or exceeds the permitted use, you will need to obtain permission directly from the copyright holder. To view a copy of this licence, visit http://creativecommons.org/licenses/by/4.0/.

\section{References}

Abram C, Fond B, Beyrau F (2015) High-precision flow temperature imaging using $\mathrm{ZnO}$ thermographic phosphor tracer particles. Opt Express 23(15):19453-19468

Abram C, Schreivogel P, Fond B, Straußwald M, Pfitzner M, Beyrau $F$ (2016) Film cooling flows using thermographic particle image velocimetry at a $6 \mathrm{kHz}$ repetition rate. In: Proceedings of 18th international symposium on the application of laser and imaging techniques to fluid mechanics, Lisbon

Abram C, Fond B, Beyrau F (2018) Temperature measurement techniques for gas and liquid flows using thermographic phosphor tracer particles. Prog Energy Combust Sci 64:93-156

Bakhtiari A, Sander T, Straußwald M, Pfitzner M (2018) Active turbulence generation for film cooling investigations. In: Proceedings of ASME turbo expo 2018, number GT2018-76451, Lillestrom (Oslo)

Bräunling W (2015) Flugzeugtriebwerke, 4th edn. Springer, Berlin

Fond B, Abram C, Heyes A, Kempf A, Beyrau F (2012) Simultaneous temperature, mixture fraction and velocity imaging in turbulent flows using thermographic phosphor tracer particles. Opt Express 20(20):22118-22133

Fond B, Abram C, Beyrau F (2015) Characterisation of the luminescence properties of BAM: $\mathrm{Eu}^{2+}$ particles as a tracer for thermographic particle image velocimetry. Appl Phys B Lasers O 121:495-509

Fond B, Xiao C-N, T'Joen C, Henkes R, Veenstra P, van Wachem BGM, Beyrau F (2018) Investigation of a highly underexpanded jet with real gas effects confined in a channel: flow field measurements. Exp in Fluids 59(160)

Fond B, Abram C, Pougin M, Beyrau F (2019) Characterisation of dispersed phosphor particles for quantitative photoluminescence measurements. Opt Mater 89:615-622

Kadotani K, Goldstein R (1979) On The nature of jets entering a turbulent flow: part A-jet-mainstream interaction. J Eng Power 101:459-465

Kakade V, Thorpe S, Gerendas M (2012), Effusion-cooling performance at gas turbine combustor representative flow conditions. In: Proceedings of ASME turbo expo 2012, number GT2012-68115, Copenhagen

Kingery J, Ames F (2015) Full coverage shaped hole film cooling in an accelerating boundary layer with high free-stream turbulence. In: Proceedings of ASME turbo expo 2015, number GT2015-42233, Montreal

Klingshirn C (2007) ZnO: material, physics and applications. ChemPhysChem 6(8):782-803

Kohli A, Bogard D (1998) Effects of very high free-stream turbulence on the jet-mainstream interaction in a film cooling flow. J Turbomach 120:785-790

Lee H, Böhm B, Sadiki A, Dreizler A (2016) Turbulent heat flux measurement in a non-reacting round jet, using BAM:Eu ${ }^{2+}$ phosphor thermography and particle image velocimetry. Appl Phys B 122(7):1-13

Ling J, Elkins C, Eaton J (2015a) Optimal turbulent schmidt number for RANS modeling of trailing edge slot film cooling. J Eng Gas Turbines Power 137:072605

Ling J, Ryan K, Bodart J, Eaton J (2015b) Analysis of turbulent scalar flux models for a discrete hole film cooling flow. In: Proceedings of ASME turbo expo 2015, number GT2015-42092, Montréal

Martin D, Thorpe S (2012) Experiments on combustor effusion cooling under conditions of very high free-stream turbulence. In: Proceedings of ASME turbo expo 2012, number GT2012-68863, Copenhagen

Melling A (1997) Tracer particles and seeding for particle image velocimetry. Meas Sci Technol 8:1406-1416

Prahl S (2018) Mie scattering calculator. https://omlc.org/calc/mie_ calc.html. Accessed 4 Oct 2020

Saumweber C, Schulz A (2012) Free-stream effects on the cooling performance of cylindrical and fan-shaped cooling holes. J Turbomach 134:061007

Saumweber C, Schulz A, Wittig S (2003) Free-stream turbulence effects on film cooling with shaped holes. J Turbomach 125:65-73

Schreivogel P, Kröss B, Pfitzner M (2014) Study of an optimized trench film cooling configuration using scale adaptive simulation and infrared thermometry. In: Proceedings of ASME turbo expo 2014, number GT2014-25144, Düsseldorf

Schreivogel P, Abram C, Fond B, Straußwald M, Beyrau F, Pfitzner M (2016) Simultaneous kHz-rate temperature and velocity field measurements in the flow emanating from angled and trenched film cooling holes. Int J Heat Mass Transf 103:390-400

Schroeder R, Thole K (2016) Effect of high freestream turbulence on flowfields of shaped film cooling holes. J Turbomach 138:091001

Schroeder R, Thole K (2017) Thermal field measurements for a shaped hole at low and high freestream turbulence intensity. J Turbomach 139:021012 
Stephan M, Lee H, Albert B, Dreizler A, Böhm B (2016) Simultaneous planar gas-phase temperature and velocity measurements within a film cooling configuration using thermographic phosphors. In: Proceedings of 18th international symposium on the application of laser and imaging techniques to fluid mechanics', Lisbon

Stephan M, Zentgraf F, Berrocal E, Albert B, Böhm B, Dreizler A (2019) Multiple scattering reduction in instantaneous gas phase phosphor thermometry: applications with dispersed seeding. Meas Sci Technol 30(5):054003

Straußwald M, Sander T, Bakhtiari A, Pfitzner M (2018) High-speed velocity measurements of film cooling applications at high-turbulence main flow conditions. In: Proceedings of ASME turbo expo 2018, number GT2018-76458, Lillestrom (Oslo)

Wieneke B (2015) PIV uncertainty quantification from correlation statistics. Meas Sci Technol 26:074002

Wright L, McClain S, Clemenson M (2010) Effect of freestream turbulence intensity on film cooling jet structure and surface effectiveness using PIV and PSP. In: Proceedings of ASME turbo expo 2010: power for land, sea and air, number GT2010-23054, Glasgow
Wright L, McClain S, Clemenson M (2011a) Effect of density ratio on flat plate film cooling with shaped holes using PSP. J Turbomach 133:041011

Wright L, McClain S, Clemenson M (2011b) Effect of freestream turbulence intensity on film cooling jet structure and surface effectiveness using PIV and PSP. J Turbomach 133:041023

Wright L, McClain S, Clemenson M (2011c) PIV investigation of the effect of freestream turbulence intensity on film cooling from fanshaped holes. In: Proceedings of ASME turbo expo 2011, number GT2011-46127, Vancouver

Wright L, McClain S, Brown C, Harmon W (2013) Assessment of a double hole film cooling geometry using S-PIV and PSP. In: Proceedings of ASME turbo expo 2013, number GT2013-94614, San Antonio

Zentgraf F, Stephan M, Berrocal E, Albert B, Böhm B, Dreizler A (2017) Application of structured illumination to gas phase thermometry using thermographic phosphor particles: a study for averaged imaging. Exp Fluids 58:82

Publisher's Note Springer Nature remains neutral with regard to jurisdictional claims in published maps and institutional affiliations.

\section{Affiliations}

\section{Michael Straußwald ${ }^{1} \cdot$ Christopher Abram ${ }^{2} \oplus \cdot$ Tobias Sander $^{1} \cdot$ Frank Beyrau $^{2} \cdot$ Michael Pfitzner $^{1}$}

Michael Straußwald

michael.strausswald@unibw.de

$\triangle$ Christopher Abram

christopher.abram@ovgu.de

1 Institute of Thermodynamics, Universität der Bundeswehr München, Werner-Heisenberg-Weg 39, 85577 Neubiberg, Germany
Institute of Fluid Dynamics and Thermodynamics, Otto-von-Guericke-Universität Magdeburg, Universitätsplatz 2, 39106 Magdeburg, Germany 